\title{
Development of LGI1 Antibody Encephalitis after Treatment of Lung Cancer
}

\author{
Suk-Won Ahn, Jeong-Min Kim, Jee-Eun Kim, Soon-Tae Lee, Dong-Won Ahn, Jung-Joon Sung
}

doi:10.1017/cjn.2014.17

Can J Neurol Sci. 2014; 41: 669-671

Autoimmune synaptic encephalitis is a disorder associated with antibodies targeting the cell surface of neurons or synaptic proteins. Neurological symptoms of autoimmune encephalitis include seizure, memory dysfunction, abnormal behavior, and cognitive impairment.

Recently, most patients with idiopathic encephalitis have been identified with autoimmune encephalitis associated with antibodies directed against the extracellular domains of cell surface proteins, which are critical in regulating neuronal excitability. ${ }^{1-4}$ Such proteins include $N$-methyl- $D$-aspartate (NMDA), $\alpha$-amino-3-hydroxy-5methyl-4-isoxazolepropionic acid (AMPA), and $\gamma$-aminobutyric acid (GABA)-B receptors, as well as the voltage-gated $\mathrm{K}^{+}$channel complex, consisting of leucine-rich, glioma-inactivated 1 (LGI1) and contactin-associated protein-like 2 (CASPR2). ${ }^{1}$

LGI1 protein has significant roles in synaptic transmission and myelination; therefore, autoantibodies against LGI1 protein would be a pathogenic cause of autoimmune encephalitis. However, the mechanism underlying LGI1 antibody encephalitis is not fully understood. Also, LGI1-antibody encephalitis has been weakly associated with cancer, in relation to paraneoplastic limbic encephalitis. ${ }^{1,2}$

Despite this weak association with cancer, the presence of LGI1 antibodies and related clinical features are significant. These conditions presenting LGI1 antibody respond well to immunotherapy and sometimes are associated with hidden malignancy. 5

Here we describe an unusual paraneoplastic case of LGI1 antibody encephalitis in a 72-year-old man presenting with symptoms of status epilepticus. Unlike previous cases of LGI1 antibody encephalitis with tumor, our patient's encephalitis developed after surgical removal of and chemotherapy for lung cancer.

\section{Case Report}

A 72-year-old man presented with cognitive impairment, followed by drug-resistant seizures. He had undergone successful treatment of lung cancer, exhibited normal behavior, and returned to work. His medical history included a diagnosis of lung cancer, verified by chest $\mathrm{CT}$, which had revealed a well-defined solitary nodule in the right upper lobe (Figure 1A). $18^{\mathrm{F}}$-fluorodeoxyglucose positron-emission tomography showed a hypermetabolic lesion in the right upper lobe, suggesting a primary lung cancer (Figure 1B). However, no metastasis or dissemination in other organs was evident. The patient therefore underwent lobectomy and wedge resection for lung cancer, and pathology had confirmed a non-small-cell lung cancer of stage IA (T1aNOM0) large-cell neuroendocrine carcinoma. After successful surgery, he was treated with cisplatin-vinorelbine combination chemotherapy without radiation, after which a chest $\mathrm{CT}$ detected no recurrence of lesions. However, after 3 months, his general activity level gradually declined and he showed neurological symptoms of language impairment and confusion, followed by tonic/dystonic seizures involving face and limbs. When he came to our department, detailed assessments of cognition, motor, and sensory function were not possible because of deteriorated mental status and sustained seizure indicating status epilepticus. Brain MRI using fluid-attenuated inversion recovery (FLAIR) and diffusionweighted images (DWI) revealed high-signal lesions in the left parieto-temporo-occipital lobe including the insula, while apparent diffusion coefficient showed isointensity or hypointensity in the lesions (Figure 2).

CSF analysis showed no leukocytes, and CSF chemistry (protein, $0.24 \mathrm{~g} / \mathrm{L}$; glucose, $3.22 \mathrm{mmol} / \mathrm{L}$ ) was normal. However, serum electrolyte test showed a low plasma sodium level of $130 \mathrm{mmol} / \mathrm{L}$.

Electroencephalography revealed distinct abnormalities of frequent medium-voltage spike and waves in right frontotemporal areas and frequent episodes of long bursts of repetitive highvoltage spike and waves suggesting electroclinical seizures.

Because of his previous lung cancer, we evaluated the chest, neck, abdomen, and pelvis CT to exclude the possibility of recurred tumor or hidden malignancy, which were all normal. We checked for antibodies, including anti-Hu, anti-Yo, anti-CV2/ CRMP5, anti-Ri, anti-Ma2, and anti-amphiphysin associated with well-known paraneoplastic limbic encephalitis. We also identified antibodies targeting neuronal cell surface antigens, ion channels (voltage-gated potassium channels), and ligand-gated ion channels (NMDA, AMPA, and GABA-B receptor channels).

Conclusively, the test for antineuronal antibodies was positive for anti-LGI1 antibody in the serum, whereas tests for other antibodies, including anti-CASPR2, were negative. The patient started treatment with $1000 \mathrm{mg}$ of methylprednisolone intravenous bolus for 5 days, followed by oral prednisolone $\left(1 \mathrm{mg} \cdot \mathrm{kg}^{-1}\right.$. day $^{-1}$ ). After high-dose steroid treatment, status epilepticus was well controlled by antiepileptic drugs, and the patient's mental and language functions gradually recovered; a follow-up MRI after high-dose steroid treatment also showed improvement.

From the Department of Neurology (SWA, JMK), Chung-Ang University Hospital, Chung-Ang University College of Medicine, Seoul, Republic of Korea; Department of Neurology (STL, JJS) and Department of Internal Medicine (DWA), Seoul National University College of Medicine, Seoul, Republic of Korea; Department of Neurology (JEK), Seoul Medical Center, Seoul, Republic of Korea.

Received January 16, 2014. Revised March 31, 2014.

Correspondence to: Suk-Won Ahn, Department of Neurology, Chung-Ang University Hospital, Chung-Ang University College of Medicine, 29 Heukseong-no, Dongjak-gu, Seoul 156-755, Republic of Korea. E-mail: icandr@hanmail.net 

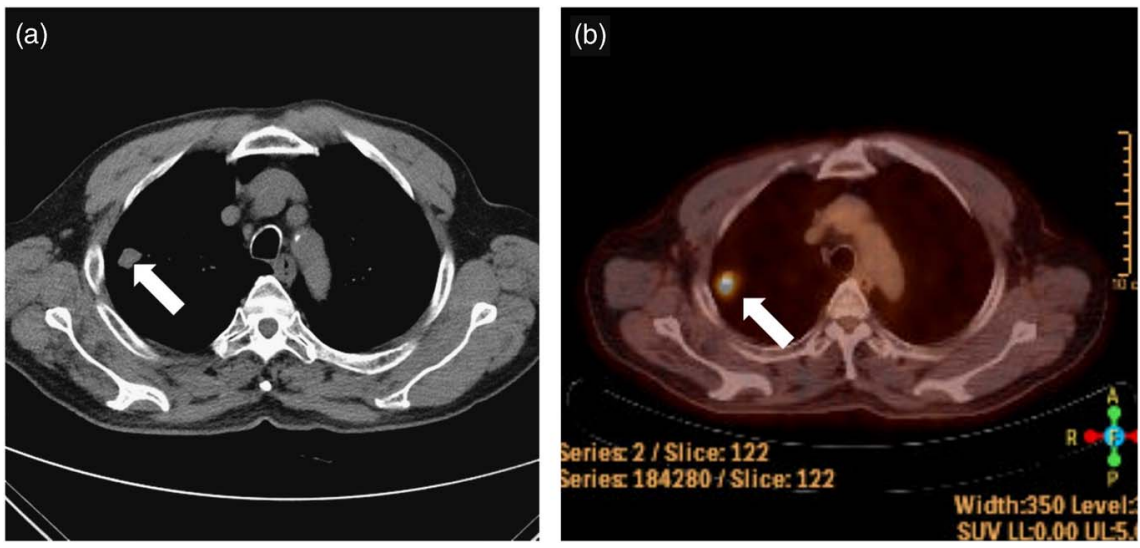

Figure 1: Chest CT scan (A) and $18^{F}$-fluorodeoxyglucose positron-emission tomography $(B)$ revealed a well-defined solitary nodule in the right upper lobe (white arrow).

\section{DISCUSSION}

We have described an unusual paraneoplastic case of postoperative LGI1 antibody encephalitis after surgical removal of and chemotherapy for lung cancer, with attention to clinical manifestations, autoimmune antibody involvement, and therapeutic effects.
Recently, researchers have identified several antibodies implicated in autoimmune encephalitis. ${ }^{1-4}$ Among them, LGI1 antibody is well established as a factor against voltage-gated potassium channels. ${ }^{4,5}$ LGI1 is a glycoprotein-secreted neuronal protein from presynaptic terminals and binds to both presynaptic A disintegrin and metalloprotease (ADAM) 23 and postsynaptic
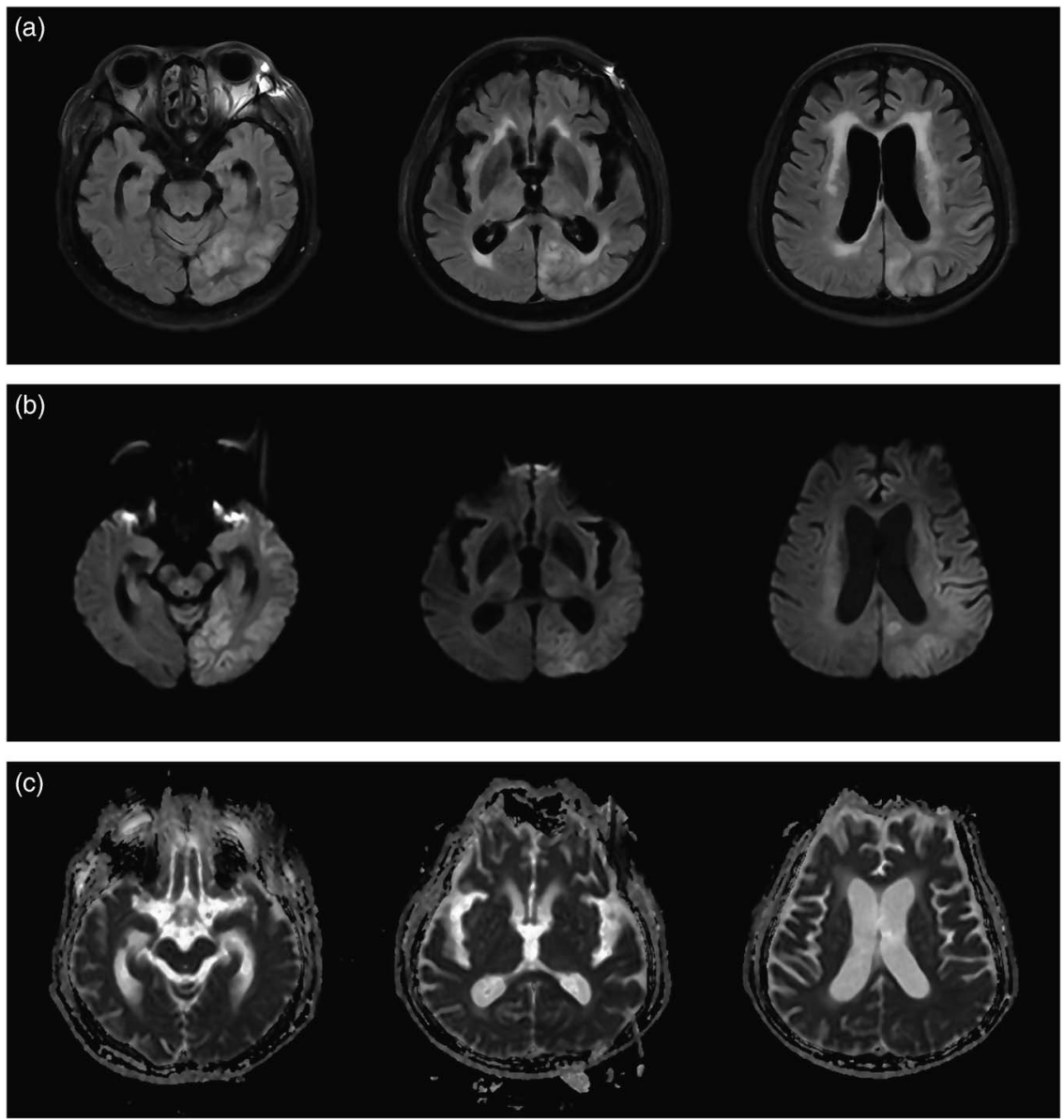

Figure 2: Brain MRI, using FLAIR (A), DWI $(B)$ and $A D C(C)$, revealed high-signal lesions without diffusion restriction in the left medial temporal and parieto-occipital lobe, including the insula. 
ADAM22. Accordingly, LGI1 may regulate synaptic strength and transmission at excitatory synapses between presynaptic kv. 1 channels and postsynaptic AMPA receptors. ${ }^{1-5}$ Therefore, autoantibody against LGI1 protein would disrupt synaptic transmission and consequently would be a pathogenic cause of autoimmune encephalitis.

The laboratory detection of antibodies and related clinical features in LGI1 antibody encephalitis can prompt early treatment and may lead to better outcomes. ${ }^{4}$ In our patient, we used an indirect immunofluorescence test to detect anti-LGI1 antibody. In brief, we reacted diluted patient serum (1:10) with HEK293 cells transfected with plasmids containing the human LGI1 sequence (Euroimmun AG, Lübeck, Germany), and we used fluorescein isothiocyanate-labeled anti-human immunoglobulin $\mathrm{G}$ as the secondary antibody. Even immunofluorescence over the cytoplasm indicated a positive reaction.

The etiology of LGI1 antibody encephalitis is not clear yet. Most patients with LGI1 antibody encephalitis did not present with tumor, so LGI1-antibody encephalitis has been only weakly associated with cancer, in relation to paraneoplastic limbic encephalitis. ${ }^{1,2}$ However, the presence of a tumor that expresses the antigenic target of the antibody can sometimes explain the LGI1 antibody. ${ }^{5}$

Therefore, from an etiologic perspective, our patient seems to have paraneoplastic autoimmune encephalitis targeting LGI1. Furthermore, the patient had been successfully treated for cancer with surgery, chemotherapy, and intermittent dexamethasone medication before LGI1 antibody encephalitis developed.

The operation or chemotherapy could have decompensated a precarious immunological homeostasis and aggravated preexisting, low-level LGI1 antibody encephalitis in our patient. In addition, he had not taken dexamethasone for 3 weeks when presenting with LGI1 antibody encephalitis. Accordingly, postoperative inflammation, chemotherapy, and withdrawal of dexamethasone might be associated with autoimmune cascade. However, we could not confirm the etiologic relevancy.

Regardless, our case has a lesson for physicians caring for patients receiving treatment for malignancy: watch for LGI1 antibody encephalitis, even if the patient is cured of cancer and returns to work. Our single case cannot clarify postoperative guidelines pertaining to LGI1 antibody encephalitis and cannot predict the necessary postsurgical observation period even given treatment responsiveness. Therefore, future case studies on postoperative LGI1 antibody encephalitis, when sufficiently extensive, may improve our understanding and clinical care of both cancer and autoimmune encephalitis.

LGI1 antibody encephalitis can develop even after successful treatment of cancer. Testing for specific autoimmune antibodies can clarify the etiology of any undetermined encephalitis or neurological symptoms accompanied by seizure.

\section{ACKNOWLEDGMents AND FUnding}

This research was supported by Basic Science Research Program through the National Research Foundation of Korea (NRF) funded by the Ministry of Science, ICT \& Future Planning (2014R1A1A1005484), and grants from the Korea Health Care Technology R\&D Project, Ministry for Health, Welfare \& Family Affairs, Republic of Korea (A110834).

\section{DisClOSURES}

The authors report no disclosures or conflicts of interest.

\section{REFERENCES}

1. Irani SR, Vincent A. Autoimmune encephalitis-new awareness, challenging questions. Discov Med. 2011;11(60):449-58.

2. Shin YW, Lee ST, Shin JW, et al. VGKC-complex/LGI1-antibody encephalitis: clinical manifestations and response to immunotherapy. J Neuroimmunol. 2013;265(1-2):75-81.

3. Lai M, Huijbers MG, Lancaster E, et al. Investigation of LGI1 as the antigen in limbic encephalitis previously attributed to potassium channels: a case series. Lancet Neurol. 2010;9(8):776-85.

4. Irani SR, Alexander S, Waters $\mathrm{P}$, et al. Antibodies to Kv1 potassium channel-complex proteins leucine-rich, glioma-inactivated-1 protein and contactin-associated protein-2 in limbic encephalitis, Morvan's syndrome and acquired neuromyotonia. Brain. 2010;133(9):2734-48

5. Lemieux J, Provencher L, Brunet D, et al. Paraneoplastic encephalomyelitis, stiff person syndrome and breast carcinoma. Can J Neurol Sci. 2011;38(5):790-2. 